\title{
Caracterización patogénica, clínica y diagnóstica de la pandemia por SARS-CoV-2
}

\author{
Pathogenic, clinical and diagnostic characteristics of the SARS-CoV-2 pandemic
}

\author{
Bastian Abarca Rozas', Jocelyn Vargas Urra² y Javier García Garzón²
}

${ }^{1}$ Facultad de Medicina, Universidad San Sebastián.

${ }^{2}$ Hospital Clínico Universidad de Chile.

Los autores declaran ausencia de conflictos de interés.

No hubo fuentes externas de financiación.

Recibido: (versión final): 24 de mayo de 2020 / Aceptado: 25 de mayo de 2020

\section{Resumen}

El SARS-CoV-2 es el agente patógeno responsable del COVID-19, enfermedad infecciosa que puede evolucionar desde un cuadro viral leve hasta la falla multiorgánica y muerte. Esta enfermedad se caracteriza por tener una tasa de transmisibilidad elevada, lo que ha permitido su diseminación por el mundo. No existen marcadores pronósticos claros que guíen la gravedad del cuadro; no obstante, hay elementos clínicos que podrían considerarse posibles predictores de gravedad. Conocer su estructura viral y patogenia ha posibilitado objetivar los pasos moleculares específicos que pueden ser blancos terapéuticos de variados fármacos, los cuales se mantienen en investigación y marcarán las directrices de futuros protocolos.

Palabras clave: SARS-CoV-2; COVID-19; enfermedad del coronavirus; enfermedad infecciosa.

\section{Introducción}

Este es un tema en evolución y, por tanto, los conceptos vertidos a continuación pueden variar en virtud de la evidencia cientifica que vaya surgiendo.

$\mathrm{E}$ 1 conocimiento del coronavirus humano existe desde el siglo XX, considerándose un agente de baja patogenicidad que generaba infecciones respiratorias leves ${ }^{1}$. En el siglo XXI surgieron dos grandes brotes de coronavirus humanos que han adquirido una patogenicidad mayor a la conocida; estos son el SARS-CoV (Severe

\begin{abstract}
SARS-CoV-2 is the pathogen responsible for COVID-19, an infectious disease that can evolve from a mild viral illness to multiple organ failure and death. This disease is characterized by a high transmissibility rate, which has lead to its spread throughout the world. There are no clear prognostic markers to guide the severity of the condition; however, some clinical elements could be considered possible predictors of severity. Knowing its viral structure and pathogenesis has allowed to recognize specific molecular pathways candidates as therapeutic targets for various drugs, which are still under investigation and will set the guidelines for future protocols.

Keywords: SARS-CoV-2; CoVID-19; coronavirus disease; infectious disease.
\end{abstract}

\section{Correspondencia a: \\ Bastian Abarca Rozas}

fds.baar@gmail.com

Acute Respiratory Syndrome-CoronaVirus) que se manifestó en China en el año 2002, alcanzando una letalidad de $10 \%{ }^{2}$, y el MERS-CoV (Middle East Respiratory Syndrome-CoronaVirus) que se presentó en Medio Oriente el año 2012, con una letalidad de 35\% ${ }^{3}$. El 30 de enero del año 2020, la Organización Mundial de la Salud (OMS) anunció un nuevo brote de coronavirus ${ }^{4}$ que rápidamente se diseminó en el mundo; declarándose el 11 de marzo del mismo año como pandemia y emergencia de salud pública.

El coronavirus 2 del síndrome respiratorio agudo severo (SARSCoV-2) es el agente responsable de causar la enfermedad del coronavirus 2019 (COVID-19) ${ }^{5}$. Este virus se originó en la ciudad de 
Wuhan (China), y posiblemente se trasmitió a partir de la ingesta de animales salvajes como pangolín, civeta de palma, serpientes, entre otros (considerados como hospedadores intermediarios donde posiblemente se desarrollaron las mutaciones y recombinaciones del virus). Los análisis de secuencia genómica han podido definir al murciélago como un probable reservorio clave, que facilitó la transmisión de este virus a los hospedadores intermediarios y, mediante el consumo de éstos, a los humanos, continuando su transmisibilidad a través de las secreciones de personas infectadas ${ }^{6}$. Esta capacidad de salto entre especies, asociada a la actual interconectividad entre países ${ }^{7}$, permitió su rápida diseminación en el mundo. Con el fin de evaluar la dinámica de transmisión del COVID-19, se utilizó el "basic reproductive ratio" o R0 (número promedio de casos nuevos que genera un caso a lo largo de un período infeccioso) necesario para conocer la velocidad de infectividad del patógeno ${ }^{8}$. En el caso del SARS-CoV el R0 era de 2,3-3,7, mientras que en el MERS-CoV el R0 fue 0,50-0,92. Si se comparan estas tasas con el actual SARS-CoV-2, éste se escapa con un R0 de 1,4-3,9, es decir, una tasa de transmisibilidad considerablemente más alta ${ }^{8}$. Estas estimaciones son susceptibles a cambios constantes (dependientes de factores biológicos, socioculturales y ambientales) a medida que esta enfermedad evoluciona, por lo que deben interpretarse con cautela ${ }^{9}$. La experiencia lograda en China concluyó que las medidas de distanciamiento social y físico posibilitaron reducir el porcentaje de R0 con el objetivo de disminuir la intensidad máxima de la pandemia; no obstante, no se tiene claridad de que estas disminuciones de R0 puedan generalizarse a otros entornos 9 . Se estima que los brotes recurrentes de SARSCoV-2 ocurren después del pico pandémico inicial; por tanto, la instauración de medidas de distanciamiento social de meses o años serían una opción para controlar la transmisión y mitigar su recurrencia. Incluso, de lograr una eliminación del virus, la vigilancia podría mantenerse por al menos cuatro años para así evitar recurrencias 9 .

\section{Etiopatogenia}

Desde la filogenética se han identificado cuatro géneros de coronavirus distintos $(\alpha, \beta, \gamma, \delta)$, siendo el SARSCoV-2 uno de los tantos $\beta$-coronavirus existentes, pero clasificado como un agente distinto y nuevo ${ }^{6,10}$. Su genoma cuenta con 32 mil pares de bases ${ }^{10}$, con una estructura molecular semejante a la de SARS-CoV y MERS-CoV, pero con una transcriptasa inversa capaz de realizar copias erróneas, que genera altas tasas de recombinación de ARN durante el período infectivo en una de sus proteínas de superficie (proteína S), logrando una mayor antigenicidad dentro del hospedero y un alto grado de transmisibilidad en comparación a la proteína S del SARS-CoV y MERS$\mathrm{CoV}^{10}$. Un estudio realizado a 4.535 genomas, que analizó el árbol filogenético del SARS-CoV-2, identificó 13 mutaciones de la proteína $\mathrm{S}$ que se están acumulando. De éstas, la mutación D614G es la forma dominante que se ha expandido desde principios de febrero ${ }^{11}$.

El conjunto de glicoproteínas que envuelven la superficie del virus le dan el aspecto típico de "corona". Una de ellas, la proteína $\mathrm{S}$ (espiga), es la encargada de unirse al receptor celular ECA-2 (enzima convertidora de angiotensina 2) en asociación a una proteasa transmembrana conocida como TMPRSS2; que favorece la endocitosis del virus (y que puede ser modulada por genes productores de andrógenos y por ciertos fármacos $)^{12}$. Se sabe que los receptores ECA-2 están ampliamente distribuidos en las vías respiratorias (incluyendo la unión bronquiolo-alveolar) y las células epiteliales alveolares, así como también en el epitelio del intestino delgado y colon, testículos, tejido adiposo, glándula tiroides, riñones, tejido miocárdico y vascular, tejido mamario, vagina, ovarios y trompas de Falopio, páncreas y en macrófagos ${ }^{12-14}$. La unión de la proteína S con los receptores ECA-2 y TMPRSS2 forma un complejo que permite la internalización del virus en la célula, liberando el genoma de ARN viral monocatenario al citoplasma. Este genoma se compone de cinco regiones genómicas, de las cuales, la región codificante de la enzima de replicación (compuesta por dos genes: ORF1a y ORF1b) y los genes estructurales ( $\mathrm{S}, \mathrm{E}, \mathrm{M}$ y N) tendrán una participación activa en la patogenia ${ }^{10}$. Los genes ORF1a y ORF1b expresan y codifican dos poliproteínas que se proteolizan en 16 subproductos (incluyendo proteasas, helicasa, exonucleasa y la ARN polimerasa dependiente de ARN o RdRp) ${ }^{1}$, los que formarán el complejo replicasatranscriptasa de ARN o transcriptasa inversa. Esta enzima se ubicará en las membranas intracelulares del retículo endoplásmico (RE) y desde aquí se iniciará la replicación, transcripción y traducción viral, codificando en parte las proteínas estructurales de espiga (S), de membrana (M), de envoltura (E) y de la nucleocápside (N). Estos subproductos virales se sumergirán en las membranas de los complejos RE y Golgi, para ser exportados de estos organelos como glicoproteínas listas para su ensamblaje. Finalmente, será por medio de vesículas que estos virus se liberarán por exocitosis para continuar infectando el resto de las células pulmonares ${ }^{15}$.

El inicio temprano de la replicación viral puede generar piroptosis y apoptosis masiva de células epiteliales y endoteliales, además de una fuga vascular e infiltración pulmonar de linfocitos; desencadenando la liberación de citocinas y quimiocinas proinflamatorias ${ }^{13}$, siendo la interleucina (IL)-6 (IL-6) el mediador principal. Este fenómeno estimulará a otras células (células B, células $\mathrm{T}$, células natural killer, células dendríticas y monocitos) a potenciar esta actividad inflamatoria, desarrollando 
el epitelio nasal podría ser dependiente de la edad. Un estudio con 305 individuos, de 4 a 60 años, mostró que la expresión del gen de la ECA-2 en el epitelio nasal fue más baja en niños menores de 10 años $(n=45)$; aumentando su expresión a medida que aumentaban en edad, elevándose a 2,77 veces en niños entre 10-17 años $(n=185), 3,02$ veces en adultos entre $18-24$ años $(n=46)$ y 3,09 veces en adultos de $\geq 25$ años $(n=29)$. En este sentido, la edad pediátrica representa $<2 \%$ de los casos identificados de enfermedad por COVID-19, presumiendo que el menor riesgo entre los niños puede deberse a esta expresión diferencial de la ECA- $2^{20}$.

La transmisión entre humanos ocurre a través del contacto cercano $(<1$ metro) con una persona con síntomas respiratorios, mediante la exposición a gotitas $(5$ a $10 \mu \mathrm{m})$ y aerosoles $(<5 \mu \mathrm{m})$ provenientes de ésta, o bien, a partir del contacto con superficies de su entorno inmediato o fómites ${ }^{21}$. Estas gotitas y aerosoles son eliminadas al hablar, toser o estornudar, incluso se ha visto que tras ciertos procedimientos se pueden generar aerosoles y facilitar la transmisión por vía aérea; entre ellos, se pueden mencionar: la intubación endotraqueal, broncoscopía, aspiración abierta, administración de un fármaco por nebulización, ventilación manual previo a la intubación, giro del paciente a decúbito prono, desconexión del paciente desde un ventilador sin clampeo del tubo endotraqueal o stand by del ventilador, ventilación no invasiva con presión positiva, traqueostomía y reanimación cardiopulmonar ${ }^{21}$. Los aerosoles pueden permanecer suspendidos por períodos de tiempo prolongados y transmitirse a distancias mayores de un metro, ingresando a través de la vía aérea o mucosas (ocular, nasal y oral) ${ }^{21}$. Inmediatamente después de que un paciente exhale las gotas cargadas con virus, el contenido líquido inicia su evaporación, generando gotitas y aerosoles tan pequeñas que el transporte por corrientes de aire afecta más que la fuerza gravitatoria, alcanzando distancias de decenas de metros desde su punto de origen ${ }^{22}$. Ante esto, se pueden establecer medidas de precaución contra la transmisión aérea, incluyendo el lavado frecuente de manos, la desinfección de la ropa protectora, el uso y desinfección adecuada de las áreas de aseo, una mayor frecuencia de ventilación de habitaciones, evitar la recirculación de aire y evitar permanecer en el flujo de aire directo de otra persona; minimizando el número de sujetos que comparten el mismo ambiente ${ }^{22}$.

Se han reportado muestras positivas tanto en saliva, orina y heces ${ }^{23,24}$, siendo estas últimas un punto importante a considerar, dado que la positividad de las muestras fecales puede durar en promedio 27,9 días desde el primer síntoma, lo que no descarta que pueda traducirse en una nueva forma de transmisión ${ }^{24}$. Al analizar tanto aerosoles como fómites, se detectó que el virus puede permanecer viable e infeccioso en aerosoles hasta por $72 \mathrm{~h}$, mientras que 
en superficies puede persistir durante días dependiendo del cobertizo del inóculo, siendo más estable en plástico $(>72 \mathrm{~h}$ ) y acero inoxidable ( $>48 \mathrm{~h}$ ) que en cobre (hasta $4 \mathrm{~h}$ ) o cartón (hasta $24 \mathrm{~h}$ ) a una temperatura ambiente de $21{ }^{\circ} \mathrm{C}$ a $23{ }^{\circ} \mathrm{C}$, con $40 \%$ de humedad relativa ${ }^{25}$. Por lo tanto, este virus puede contagiar por contacto directo con una persona infectada $y$, de forma indirecta, por contacto con superficies de su entorno inmediato o con objetos que haya utilizado. No obstante, es posible su inactivación en presencia de etanol al $95 \%$ o de hipoclorito sódico en concentraciones de $0,1 \%{ }^{26}$ como medidas de prevención y control.

El período de incubación se define como el período de tiempo desde que alguien se infecta hasta que se desarrolla las manifestaciones clínicas, con una media aproximada de 4-5 días, pudiendo manifestar síntomas entre el día 2 y 14 en $97,5 \%$ de los $\operatorname{casos}^{27}$, de los cuales, $5 \%$ la desarrollará el día 2, 50\% dentro de los 5 días después de la infección y $95 \%$ la habrá desarrollado a los 14 días $^{28}$ y menos de $3 \%$ a partir de 15 o más días ${ }^{25}$. El pico de la carga viral se alcanza dentro de los primeros 5 días, definiendo un período de tiempo en el que una persona infectada puede transmitir el virus a otros (período infeccioso), lo que se inicia antes de la fase sintomática (24-48 h previas) $)^{29,30}$ y puede perdurar durante 7-12 días en casos leves a moderados, o bien, durante más de 2 semanas en casos severos. Incluso, pacientes recuperados pueden mantener una rRT-PCR (reacción en cadena de la polimerasa en tiempo real con transcriptasa inversa) positiva durante dos semanas posteriores a la resolución de sus síntomas ${ }^{29}$, sin presentar deterioro clínico, ni asociarse a contagio de personas en contacto, debido a las bajas concentraciones de material viral detectado ${ }^{31}$.

No obstante, esta rRT-PCR positiva tardía no aclara la posibilidad de una reinfección o de un estado de portador viral, dado que este examen no es sinónimo de viabilidad, pudiendo persistir positiva por períodos superiores al período de contagiosidad del paciente ${ }^{32}$. Es importante considerar que los sujetos asintomáticos también tienen un período infeccioso activo, pero cuya temporalidad es difícil definir. Ante esto, se ha asumido este período de tiempo en torno a los 10 a 14 días desde la confirmación del caso con un test diagnóstico.

\section{Manifestaciones clínicas}

En la mayoría de los casos, las manifestaciones clínicas son leves y auto-limitadas ${ }^{23}$; sin embargo, pacientes con co-morbilidades asociadas (hipertensión arterial, enfermedades cardiovasculares y cerebrovasculares, diabetes mellitus, enfermedad pulmonar obstructiva crónica, asma bronquial alérgica, obesidad, enfermedad inflamatoria intestinal, enfermedad hepática crónica, enfermedad renal crónica, cáncer o inmunodeficiencias) o con tabaquismo activo pueden aumentar el riesgo de enfermedad grave o ser un indicador pronóstico negativo, respectivamente ${ }^{33,34}$. Un estudio identificó como factor de riesgo importante la edad de las personas infectadas, alcanzando una mayor mortalidad en personas de 85 años o más (10-27\%), seguido de los grupos entre 65 a 84 años (3-11\%), 55 a 64 años (1-3\%), y menores de 54 años $(<1 \%)^{35}$. Se ha observado una mayor frecuencia de infección en hombres mayores con co-morbilidades respecto a otros grupos ${ }^{34}$. Por otro lado, los pacientes con hipertensión arterial han mostrado una frecuencia mayor de infección, la que se desconoce si tiene relación directa con el uso de IECA ${ }^{25}$. No obstante, a la fecha no se han publicado estudios que demuestren esta asociación, por lo que no es posible establecer con seguridad si los IECA aumentan o disminuyen el riesgo de infección.

Desde el punto de vista patogénico, esta enfermedad puede entenderse a través de dos fases: la primera desencadenada por el virus y la segunda desencadenada por la respuesta del hospedero. Para facilitar aún más la comprensión de su expresión clínica, se ha clasificado su evolución en tres etapas consecutivas y superpuestas que van desde la más leve a la más grave. Esta clasificación ha permitido integrar hallazgos clínicos, respuesta a la terapia y evolución clínica (Tabla 1).

\section{Etapa I (leve)}

Ésta incluye el período de inoculación viral y el establecimiento temprano de la enfermedad. En esta fase predominan los síntomas gripales, los que pueden variar desde un cuadro subagudo a uno agudo, con síntomas leves e inespecíficos, incluyendo malestar general, fiebre y tos seca como síntomas más frecuentes ${ }^{33,34,36}$, pudiendo ser precedidos por episodios aislados de diarrea ${ }^{33,34}$. Otras de las manifestaciones clínicas observadas son disnea, mialgias y/o fatiga, astenia, cefalea, congestión nasal, expectoración, dolor faríngeo, conjuntivitis, disgeusia

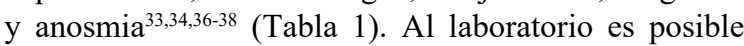
observar linfocitosis, linfopenia y alteración de marcadores inflamatorios: proteína $\mathrm{C}$ reactiva $(\mathrm{PCR})$, tiempo de protrombina, dímero $\mathrm{D}$, y lactato deshidrogenasa o $\mathrm{LDH}^{36}$. La recuperación suele ser espontánea en la mayoría de las personas con síntomas leves ${ }^{23}$; sin embargo, la enfermedad puede complicarse y progresar.

\section{Etapa II (moderada)}

Durante esta etapa, los pacientes persisten con tos y fiebre, pero desarrollan una neumonía viral ${ }^{33}$ que puede alcanzar un mayor compromiso pulmonar, caracterizado por ausencia de hipoxia (Etapa IIa) o evolucionar con hipoxia (definida como $\mathrm{PaO}_{2} / \mathrm{FiO}_{2}<300 \mathrm{mmHg}$ ) (Etapa $I I b)^{33,36}$. Al estudio se suma la alteración de las enzimas hepáticas (transaminitis) y la alteración de las imágenes torácicas (Tabla 2$)^{36}$. 
Tabla 1. Manifestaciones clínicas y principales complicaciones de COVID-19 en adultos

\section{Generalidades}

- Período de incubación promedio: 5 días

- Manifestaciones clínicas entre el día 2 y día 14, con una media de 5 días

- Tasa de transmisibilidad: 1,4-3,9\%

- Promedio de edad de los afectados: 55,5 años

\section{Complicaciones clínicas}

- Disfunción miocárdica y falla cardíaca

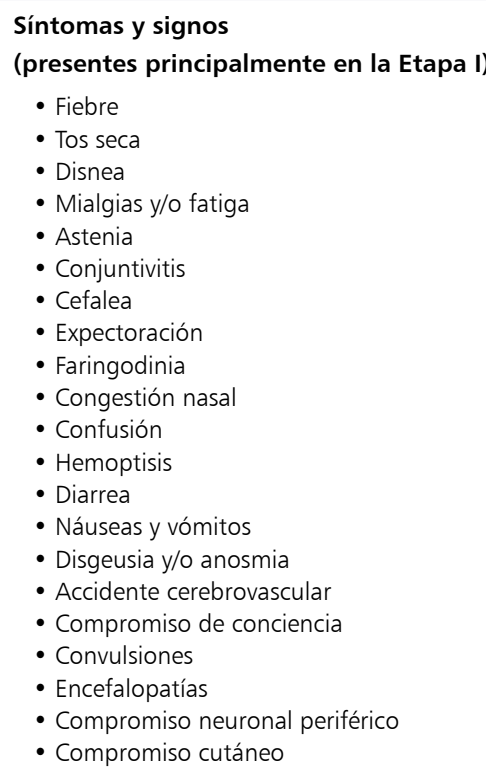

- Tos seca

- Disnea

- Mialgias y/o fatiga

- Astenia

- Conjuntivitis

- Cefalea

- Expectoración

- Faringodinia

- Congestión nasal

- Confusión

- Hemoptisis

- Diarrea

- Náuseas y vómitos

- Disgeusia y/o anosmia

- Accidente cerebrovascular

- Compromiso de conciencia

- Convulsiones

- Encefalopatías

- Compromiso neuronal periférico

- Compromiso cutáneo

- Neumonía

- SDRA

- Necesidad de ventilación mecánica

- Shock séptico

- Insuficiencia renal aguda

- Coagulopatías

- Compromiso cutáneo

- Compromiso neurológico

- Necesidad de diálisis

- Necesidad de circulación extracorpórea

- Muerte

Frecuencia

(\%)

$43-98$

$48-82$

$18-70$

$11-44$

38

$0,8-31,6$

$8-28$

$7-28$

$5-23$

$4-14$

9

5

$2-4$

$1-3$

- - -

- - -

- - -

$-\cdots$

$-{ }^{-}-$

$-\cdots$

$-\cdots$

Etapa Frecuencia

(\%)

$\begin{array}{cc}\text { Ila } & 80-91 \\ \text { IIb - II } & 3-42 \\ \text { IIb - III } & 6,1-17 \\ \text { III } & 6,1-20 \\ \text { III } & 7-23 \\ \text { III } & 0,5-15 \\ \text { III } & 0,1-19 \\ \text { III } & --- \\ \text { III } & --- \\ \text { III } & 0,8-9 \\ \text { III } & 0,5-3 \\ & 1,4-28,3\end{array}$

Elaboración propia $8,27,33,34,36-43,59$.
Tabla 2. Laboratorio e imágenes

\section{Laboratorio general Comentario}

- Hemoglobina

- Leucocitosis

- Leucopenia

- Plaquetas

- Trombocitopenia

- IL-6

- VHS

- Albúmina

- Enzimas hepáticas

- Bilirrubina total

- Glicemia

- Creatinina

- CK

- LDH

- PCR

- Procalcitonina

- Ferritina sérica

- NT-Pro-BNP

- hs-cTnl

- Dímero D

- Tiempo de protrombina

- Fibrinógeno

Normal-Anemia

Predominio neutrofílico (frecuente en SDRA)

Casos graves

Recuento normal (casos leves)

Probable indicador de gravedad

Aumentada en casos graves (marcador pronóstico)

Aumentada en la mayoría de los casos

Disminuida en la mayoría de los casos

Aumentada en estados pro-inflamatorios

Normal o aumentada en estados pro-inflamatorios

Aumentada en casos graves

Aumentada en casos graves

Aumentada en casos graves

Aumentada en SDRA (marcador pronóstico)

Aumentada en la mayoría de los casos (marcador de gravedad)

Aumentada en casos graves

Aumentada en casos graves (marcador pronóstico)

Aumentada en casos graves

Aumentada en casos graves (marcador pronóstico)

Aumentada en casos graves (marcador pronóstico)

Aumentada en casos graves (marcador pronóstico)

Aumentada en casos graves (marcador pronóstico)

\section{Estudio etiológico}

- $r R T-P C R$

- Test de detección de anticuerpos totales por ELISA (IgM e IgG)

- Test de diagnóstico rápido (RDT) y test de detección de anticuerpos neutralizantes

\section{Técnica}

Radiografía de

tórax

$\mathrm{TC}$

\section{Característica}

- Vidrio esmerilado

- Patrón en parche local

- Patrón en parche bilateral

- Patrón intersticial

- Vidrio esmerilado (periférico, posterior, inferior)

- Áreas de consolidación

- Patrón intersticial (con engrosamiento septal)

- Dilatación bronquial y engrosamiento de pared

- Patrón en parche bilateral

- Patrón en parche local
Frecuencia (\%)

20
28
37
4
$56-77$
$55-78$
$14-75$
55,6
52
42

Abreviaturas: CK: creatina quinasa; hs-cTnl: troponina I de alta sensibilidad; IL-6: interleuquina 6; LDH: lactato deshidrogenasa; NT-Pro-BNP: porción N-terminal del pro-péptido natriurético tipo $B$; PCR: proteína $C$ reactiva; rRT-PCR: reacción en cadena de polimerasa en tiempo real con transcriptasa inversa; TC: tomografía computada; VHS: velocidad de eritrosedimentación. Elaboración propia ${ }^{32,33,36,39,40,49,50,52,57}$.

\section{Etapa III (grave)}

Se manifiesta por un estado inflamatorio secundario al SLC. Esta fase se caracteriza por un aumento exacerbado de las proteínas de fase agudas y de los biomarcadores inflamatorios. Puede cursar con FMO, que rápidamente evoluciona a shock, vasoplejia, SDRA y colapso cardiopulmonar ${ }^{33,36}$. Se caracteriza por una elevación significativa de los biomarcadores inflamatorios (los mismos de la etapa I, a los que se suman IL-6 y ferritina sérica) y de

disfunción endotelial (troponina I ultra sensible o hs-cTnI, y la porción N-terminal del pro-péptido natriurético tipo B o NT-proBNP) (Tablas 1 y 2) ${ }^{36}$. Las manifestaciones pro-coagulantes, destacan por una marcada trombosis microvascular pulmonar difusa y por hemorragias asociadas a la inflamación alveolar e intersticial extensa, reflejadas con el aumento de las concentraciones circulantes de dímero D (que refleja la trombosis del lecho vascular pulmonar con fibrinólisis) y las elevadas concentraciones 
de hs-cTnI y NT-proBNP (que reflejan la disfunción ventricular emergente, secundaria a un aumento de la resistencia vascular pulmonar) $)^{39}$. Por otro lado, para objetivar el impacto cardiovascular, un estudio incluyó 2.736 pacientes con COVID-19, comparando el riesgo de mortalidad versus niveles de hs-cTnI las primeras $24 \mathrm{~h}$. Alrededor de $40 \%$ presentó concentraciones plasmáticas elevadas de hs-cTnI, concluyendo que, ante rangos de hs-cTnI entre 0,03-0,09 ng/mL, se tuvo un riesgo de mortalidad de 1,77 veces (IC 95\% 1,39-2,26); mientras que con concentraciones mayores de hs-cTnI ( $>0,09 \mathrm{ng} /$ $\mathrm{dL}$ ), el riesgo fue de 3,23 veces (IC 95\% 2,59-4,02) . $^{40}$.

Las manifestaciones oculares también han sido un foco clave en esta infección. En un estudio con 38 pacientes, un total de 12 sujetos $(31,6 \%)$ presentaron manifestaciones oculares consistentes con conjuntivitis (hiperemia conjuntival, quemosis, epífora o secreciones aumentadas). De éstos, se detectó rRT-PCR positiva en hisopados nasofaríngeos en 11 de estas personas, detectando además una rRT-PCR positiva en la conjuntiva ocular en dos de estos 11 pacientes ${ }^{41}$.

En relación al compromiso olfatorio y del gusto; esto ha abierto el foco de atención en cuanto al compromiso neurológico que pueda desarrollarse en contexto de la infección por SARS-CoV-2. Se han documentado (además de la cefalea, la anosmia y la ageusia) episodios de enfermedades cerebrovasculares agudas, compromiso de conciencia, convulsiones, encefalopatías y compromiso neuronal periférico ${ }^{42,43}$. Un reciente estudio propone como hipótesis la participación de receptores NRP1 (neuropilina-1) en asociación al ECA-2 y TMPRSS2, dado que su expresión fue detectada en células endoteliales (presentes en cerebro, bulbo olfatorio y tractos nerviosos), células pulmonares y en epitelio olfatorio, observando además que la expresión de estos tres receptores al mismo tiempo generaba una infección por SARS-CoV-2 más potente ${ }^{44}$. Si bien, hace falta más investigación al respecto, no se descarta la posibilidad de ampliar el entendimiento de la patogenia de este virus.

Por otro lado, se han registrado hallazgos de compromiso dermatológico presentes, tanto en la etapa I como en la etapa III, los que podrían resumirse en exantema maculopapular (47\%), lesión urticarial (19\%), áreas acrales de eritema con vesículas o pústulas (pseudosabañón) (19\%), exantema vesicular (9\%), y livideces o necrosis $(6 \%)^{45,46}$. Es frecuente ver pseudo-sabañones una vez desarrollada gran parte de la enfermedad, versus el exantema vesicular que se ve con mayor frecuencia de forma previa al inicio de los síntomas respiratorios ${ }^{46}$. Pese a ello, no existen registros que asocien estos hallazgos con la gravedad de la enfermedad, además de que la evidencia disponible es escasa.

A diferencia de los adultos, los pacientes pediátricos no parecen tener un mayor riesgo de enfermedad por
COVID-19 (confirmándose en $<2 \%$ de los menores de 18 años $)^{20}$, mostrando buen pronóstico y una rápida recuperación en $<2$ semanas luego del inicio del cuadro ${ }^{47}$. Se cree que la adquisición del virus se da principalmente a través del contacto cercano con sus padres u otros miembros de la familia $^{47}$. Su clínica se correlaciona con las fases I, II y III (descritas previamente), pero en porcentajes distintos a los vistos en la edad adulta (tanto en frecuencia como en intensidad de los síntomas), destacando la fiebre (36-60\%), tos (19-65\%), congestión faríngea u odinofagia (3-15\%), fatiga $(15 \%)$, rinorrea $(15 \%)$, taquipnea $(10 \%)$, disnea (3\%), neumonía (53\%) o cuadros asintomáticos $(28 \%)^{48,49}$. Pacientes con $<1$ año de vida, y con co-morbilidades, pueden desarrollar cuadros clínicos más graves ${ }^{48}$; no obstante, no se dispone de datos actuales que asocien el rol de las co-morbilidades con la gravedad del COVID-19 pediátrico. Una vez instalada la fase inflamatoria y procoagulante (Fase III), se ha descrito el desarrollo de un cuadro con características similares a vasculitis de Kawasaki (síndrome de shock de la enfermedad de Kawasaki o síndrome de shock tóxico), presentando fiebre, exantema, conjuntivitis, edema periférico, dolor generalizado en extremidades y síntomas gastrointestinales, evolucionando a un shock vasopléjico refractario, asociado a pequeños derrames pleurales, pericárdicos y ascíticos, posiblemente en contexto del SLC ${ }^{50,51}$. Si bien, esta clínica sólo se ha visto en reportes de casos, no se descarta la posibilidad de confirmar su asociación con SARS-CoV-2 a partir de la evidencia que surja.

\section{Diagnóstico}

\section{Detección viral}

El estándar de oro para el diagnóstico de COVID-19 sigue siendo la prueba de rRT-PCR realizada con hisopado nasofaríngeo $\mathrm{u}$ otras muestras del tracto respiratorio superior (torunda de garganta o de saliva). Una sola muestra nasofaríngea basta para hacer el diagnóstico ${ }^{52}$ y tarda en promedio 4-6 h en generar el resultado. En la mayoría de los infectados sintomáticos, la positividad de la prueba ocurre desde el primer día de los síntomas, alcanzando su máximo dentro de la primera semana y, a partir de la tercera semana, inicia su descenso hasta volverse indetectable. En pacientes hospitalizados graves, puede persistir positiva más de tres semanas después del inicio de la enfermedad ${ }^{32}$. Esta positividad disminuye más lentamente en el esputo y aún puede ser positiva después de que los hisopados nasofaríngeos sean negativos. Esta detección se ha visto en $93 \%$ de las muestras de lavado broncoalveolar, $72 \%$ de esputo, $63 \%$ de torunda nasal y $32 \%$ de torunda faríngea; incluso, $57 \%$ de los infectados mostró positividad de la RPC en heces, la que se mantuvo positiva más allá del hisopado nasofaríngeo en una 


\section{Test diagnósticos rápidos}

Detección del virus. Los test de diagnóstico rápido (RDT) reflejan la presencia o ausencia (cualitativa) de antígenos propios del SARS-CoV-2. Se cree que pueden tener una buena correlación con la rRT-PCR; tardando entre 15-20 min en mostrar resultados (positivo o negativo). Se han desarrollado y comercializado ampliamente, pero son de calidad variable $e^{55}$, ya que poseen una baja sensibilidad (aunque un valor predictor positivo alto) lo que podría llevar a que un porcentaje importante de sujetos infectados no logren ser pesquisados. Estos antígenos se detectan sólo si el virus se encuentra replicándose de forma activa, lo que otorga utilidad en la detección temprana o aguda de esta infección ${ }^{55}$. Pueden usar muestras de saliva o fluidos de hisopos nasales. Se describen falsos positivos por cruce antigénico con coronavirus causantes de infecciones respiratorias alta de curso benigno.

La OMS NO recomienda actualmente los test rápidos para la detección de antígenos en el uso clínico, aunque estimula investigar su evaluación y futura validación.

Detección de anticuerpos. En sangre se pueden pesquisar anticuerpos tipo IgM e IgG de personas supuestamente infectadas con el SARS-CoV-2. Se han desarrollado kits utilizando reacciones de inmunocromatografía y quimioluminescencia. Si bien su sensibilidad es elevada ( $>90 \%)$, su utilidad clínica en la toma de decisiones con un caso sospechoso de COVID-19 es limitada, como toda técnica serológica, debido a la latencia superior a 7-10 días en ser detectables los anticuerpos. Al igual como sucede con las técnicas rápidas de detección de antígenos, se describen falsos positivos por cruce antigénico con coronavirus causantes de resfrío común.

La OMS NO recomienda actualmente los test rápidos para la detección de anticuerpos en el uso clínico, aunque estimula investigar su evaluación y futura validación con fines clínicos y epidemiológicos.

\section{Interpretación de la respuesta inmune}

Como se comentó, hay pacientes que continúan dando positivo para SARS-CoV-2 luego de recuperarse (sea por recaída o reinfección) $)^{56}$, por lo que, la duración de eliminación del virus es un concepto incierto, siendo detectado ARN viral durante días o semanas después de la recuperación (aún a concentraciones bajas y en ausencia de síntomas) ${ }^{57}$. En este sentido, se ha planteado la hipótesis de una posible inmunidad residual temporal, conferida luego de la fase de recuperación (probablemente contra la reinfección o para disminuir la gravedad de ésta $)^{57}$. Si bien, sólo ha sido posible obtener resultados a partir de estudios en animales, que han confirmado la presencia de respuestas inmunes con eficacia protectora contra el SARS-CoV-2 ${ }^{58}$, es incierto que la detección de anticuerpos (IgM, IgG y neutralizantes) pueda asociarse a 
Tabla 3. Diagnósticos

\section{Caso sospechoso}

- Fiebre $>37,8^{\circ} \mathrm{C}$ (sin otra causa evidente)

- Síntomas sugerentes de infección respiratoria (ver Tabla 1) y/o anosmia o disgeusia

\section{Caso probable}

- Caso sospechoso con pruebas de laboratorio inconclusas

- Caso sospechoso que no pudo realizarse las pruebas de laboratorio

\section{Caso confirmado}

- Caso sospechoso con pruebas de laboratorio positivas y/o imágenes características

\section{Paciente contacto}

Persona que experimentó una exposición durante los dos días previos y los 14 días posteriores al inicio de los síntomas de un caso probable o confirmado. Se define exposición como:

- Contacto con caso probable o confirmado a $<1$ metro y durante $>15 \mathrm{~min}$

- Contacto físico directo con un caso probable o confirmado

- Atención directa a un paciente con COVID-19 probable o confirmado, sin usar el equipo de protección personal adecuado

Elaboración propia ${ }^{59,62}$ más altas que la de los pacientes sobrevivientes ${ }^{53}$. En la práctica clínica, el uso de dímero $\mathrm{D}>1 \mu \mathrm{g} / \mathrm{ml}, \mathrm{LDH}$ $>250 \mathrm{U} / \mathrm{L}$, ferritina $>600 \mu \mathrm{g} / \mathrm{L}$ e IL-6 elevada (3 o más veces su límite superior normal) pueden ser marcadores estimativos de un SLC en curso. La edad se considera un factor pronóstico importante ${ }^{35}$; donde bajo los 50 años la mortalidad es poco frecuente, menor a $2 \%$. Sin embargo, a partir de los 50 años, por cada década cumplida, la mortalidad se dobla en porcentaje (alcanzando hasta $16 \%$, a partir de los 80 años) $)^{53}$. Por otro lado, llama la atención la elevación de la procalcitonina en los pacientes pediátricos, lo cual no es común en adultos ${ }^{49}$, además de la co-infección pulmonar con otros patógenos (citomegalovirus, influenza, micoplasma y virus respiratorio sincicial $)^{49}$ que se ha observado en los niños.

\section{Imagenología}

La imagenología es un complemento necesario para entender los cambios expresados en la etapa II. Un estudio comparó la frecuencia de hallazgos anormales en la radiografía de tórax versus la tomografía computada (TC) de tórax, variando de 59,1 a 86,2\%, respectivamente ${ }^{33}$, haciendo preferible la TC como estudio imagenológico. De los hallazgos más frecuentes, se pueden mencionar el patrón en vidrio esmerilado, focos de consolidación, reticulación o tabiques interlobulares engrosados (infiltrado alveolar e intersticial) y/o presencia de nódulos pulmonares, hallazgos que varían de unilaterales a bilaterales (Tabla 2) ${ }^{60}$. Otro estudio, con 1.014 casos, correlacionó las pruebas de TC de tórax y rRT-PCR para COVID-19 en China, concluyendo que la TC de tórax tiene una sensibilidad de $97 \%$ para el diagnóstico de esta enfermedad. Del total de casos estudiados, $59 \%$ mostró resultados positivos de rRT-PCR y $88 \%$ con TC de tórax positivas, dando con ello un alto valor predictor. De los casos con resultados de rRT-PCR negativos, 75\% mostró hallazgos positivos en la TC de tórax, de los cuales $48 \%$ se consideró como casos altamente probables y $33 \%$ como caso probable (que más tarde se confirmarían con positividad de la rRT-PCR). Incluso, 42\% mostró una mejoría de las TC de tórax de seguimiento antes de que los resultados de rRT-PCR se volvieran negativos ${ }^{60}$; no obstante, no se ha analizado el impacto secundario a la radiación. Pese a la evidencia que apoya el uso de TC como principal técnica de estudio, la ecografía pulmonar es también una herramienta útil que podría adelantar la toma de decisiones, mostrando hallazgos imagenológicos compatibles con la clínica instalada. Un estudio realizado a 20 pacientes con COVID-19 (4 casos moderados, 5 graves y 11 críticos), mostró que $100 \%$ de éstos presentó hallazgos anormales en la ecografía, destacando la detección de líneas B (100\%), anomalías de la línea pleural $(100 \%)$ y focos de consolidación pulmonar (64\%), encontrando además, y de forma exclusiva en los pacientes críticos, derrame 
pleural (18\%), derrame pericárdico $(9 \%)$ y trombosis venosa profunda $(64 \%)^{61}$.

\section{Pronóstico y evolución}

Aproximadamente, $81 \%$ de los adultos sintomáticos cursan con una infección leve o moderada, $14 \%$ con infección grave y $5 \%$ con infección crítica. Se estima que van a evolucionar a enfermedad crítica entre $10-15 \%$ de los cuadros leves a moderados y entre un $15-20 \%$ de los cuadros graves. De este modo, el porcentaje de recuperación será de $96 \%$ y $4 \%$ fallecerá (con una mortalidad de $50 \%$ de los pacientes críticos) $)^{27,62}$ en un tiempo estimado de 14 días $^{25}$. De los pacientes que cursan con una infección de intensidad leve, éstos manifestarán cuadros sin neumonías ni complicaciones; no obstante, un porcentaje de éstos tendrá deterioro clínico a los 7 días de iniciada la manifestación clínica, en especial aquellos con factores de riesgo que requieren de una vigilancia médica más estricta $^{63}$. Las infecciones moderadas manifestarán clínica de neumonía asociada a disnea, la que puede evolucionar a un estadio de mayor gravedad y que actualmente se tiene definida en dos patrones fenotípicos distintos: Fenotipo L y Fenotipo $\mathrm{H}^{64}$. En el caso del fenotipo L, es posible estimar un parénquima denso con la TC, con infiltrados parciales de tipo vidrio esmerilado, una alta compliance y poco reclutables, caracterizados por su pobre oxigenación (debido al compromiso ventilación-perfusión secundario al daño endotelial y a la pérdida de la vasoconstricción hipóxica) y mayor riesgo de sobre-distensión. Por otro lado, en el caso del fenotipo $\mathrm{H}$, son pulmones con focos de consolidación extensos a la imagenología, con una mayor densidad estimada por TC, caracterizados por una baja compliance, una mejor respuesta a estrategias ventilatorias protectoras y mayor reclutabilidad; muy semejante a los SDRA habituales ${ }^{64}$.

\section{Conclusiones}

El SARS-CoV-2 es el agente responsable de causar el COVID-19, el que ha logrado una rápida diseminación a nivel mundial gracias a su alta tasa de transmisibilidad.

Tiene un período de incubación de cinco días y puede hacerse sintomático entre los días 2 y 14, manifestándose principalmente con fiebre y tos.

Aproximadamente, $81 \%$ de los pacientes adultos muestra un cuadro de intensidad leve a moderada y solo 5\% alcanza una intensidad crítica con necesidad de manejo intensivo.

Pese a no aclararse aún los factores pronósticos de estos pacientes, se puede considerar la edad avanzada, una puntuación SOFA alta y un dímero $\mathrm{D}>1 \mu \mathrm{g} / \mathrm{ml}$ como posibles predictores de gravedad.

El conocimiento de su patogenia permitirá un tratamiento dirigido contra los mecanismos moleculares del SARS-CoV-2, que de momento no han logrado consensuar con resultados prometedores o mayor efectividad terapéutica. No obstante, siguen en curso ensayos y estudios que probablemente cambien el curso de los manejos actuales; por cual, las medidas de aislamiento y cuarentena son la única medida efectiva que podría lograr disminuir la transmisibilidad del SARS-CoV-2, asociado al uso de los elementos de protección personal, manteniendo el manejo sintomático y de soporte como la indicación vigente de los pacientes infectados.

\section{Referencias bibliográficas}

1.- Weiss S R, Leibowitz J L. Coronavirus pathogenesis. In: Advances in Virus Research [Internet]. Academic Press Inc.; 2011. p. 85164. Available from: https://www.ncbi.nlm.nih. gov/pmc/articles/PMC7149603/ [citado el 6 de mayo de 2020].

2.- $\mathrm{WHO} \mid$ Overview [Internet]. Available from: https://www.who.int/whr/2003/overview/en/ index3.html [citado el 6 de mayo de 2020].

3.- Middle East Respiratory Syndrome Coronavirus (MERS-CoV) [Internet]. Available from: https://www.who.int/en/news-room/factsheets/detail/middle-east-respiratory-syndromecoronavirus-(mers-cov) [citado el 6 de mayo de 2020].

4.- WHO Director-General's opening remarks at the media briefing on COVID-19 - 11 March 2020 [Internet]. Available from: https://www. who.int/dg/speeches/detail/who-directorgeneral-s-opening-remarks-at-the-media- briefing-on-covid-19-11-march-2020. [citado el 6 de mayo de 2020].

5.- Zhu N, Zhang D, Wang W, Li X, Yang B, Song $\mathrm{J}$, et al. A novel coronavirus from patients with pneumonia in China, 2019. N Engl J Med [Internet]. 2020; 382 (8): 727-33. Available from: http://www.nejm.org/doi/10.1056/ NEJMoa2001017 [citado el 6 de mayo de 2020].

6.- Shereen M A, Khan S, Kazmi A, Bashir N, Siddique R. COVID-19 infection: Origin, transmission, and characteristics of human coronaviruses. J Adv Res [Internet]. 2020; 24: 91-8. Available from: https://www. sciencedirect.com/science/article/pii/ S2090123220300540 [citado el 6 de mayo de 2020].

7.- HAN Archive-00426 | Health Alert Network (HAN) [Internet]. Available from: https:// emergency.cdc.gov/han/han00426.asp [citado el 6 de mayo de 2020].

8.- Sun J, He W-T, Wang L, Lai A, Ji X, Zhai X, et al. COVID-19: Epidemiology, evolution, and cross-disciplinary perspectives. Trends Mol Med [Internet]. 2020; 26 (5): 483-95. Available from: https://linkinghub.elsevier.com/retrieve/ pii/S1471491420300654 [citado el 6 de mayo de 2020].

9.- Kissler S M, Tedijanto C, Goldstein E, Grad Y H, Lipsitch M. Projecting the transmission dynamics of SARS-CoV-2 through the postpandemic period. Science (80-) [Internet]. 2020; 368 (6493): 860-8. Available from: https://www.sciencemag.org/lookup/ doi/10.1126/science.abb5793 [citado el 6 de mayo de 2020].

10.- Li X, Geng M, Peng Y, Meng L, Lu S. Molecular immune pathogenesis and diagnosis of COVID-19. J Pharm Anal [Internet]. 2020; 10 (2): 102-8. Available from: https:// linkinghub.elsevier.com/retrieve/pii/ S2095177920302045 [citado el 6 de mayo de 2020].

11.- Korber B, Fischer W, Gnanakaran S G, Yoon 


\section{—}

$\mathrm{H}$, Theiler J, Abfalterer W, et al. Spike mutation pipeline reveals the emergence of a more transmissible form of SARS-CoV-2. bioRxiv [Internet]. 2020;2020.04.29.069054. Available from: https://www.biorxiv.org/content/10.1101/ 2020.04.29.069054v2.

12.- Lukassen S, Chua R L, Trefzer T, Kahn N C, Schneider M A, Muley T, et al. $<$ scp $>$ SARS $<$ I scp $>-\mathrm{CoV}-2$ receptor $<\mathrm{scp}>\mathrm{ACE}</ \mathrm{scp}>2$ and $<$ scp $>$ TMPRSS $<$ scp $>2$ are primarily expressed in bronchial transient secretory cells. EMBO J [Internet]. 2020; 39 (10): 1-15. Available from: https://www.ncbi.nlm.nih.gov/ pmc/articles/PMC7232010/

13.- Fu Y, Cheng Y, Wu Y. Understanding SARSCoV-2-mediated inflammatory responses: from mechanisms to potential therapeutic tools. Virol Sin [Internet]. 2020; 1-6. Available from: https://www.ncbi.nlm.nih.gov/pmc/articles/ PMC7090474/ [citado el 6 de mayo de 2020].

14.- Chen L, Li X, Chen M, Feng Y, Xiong C. The ACE2 expression in human heart indicates new potential mechanism of heart injury among patients infected with SARS-CoV-2. Cardiovasc Res [Internet]. 2020; 116 (6): 1097 100. Available from: https://academic.oup.com/ cardiovascres/article/116/6/1097/5813131.

15.- de Wit E, van Doremalen N, Falzarano D, Munster V J. SARS and MERS: recent insights into emerging coronaviruses. Nat Rev Microbiol [Internet]. 2016; 14 (8): 523-34. Available from: http://www.nature.com/articles/ nrmicro.2016.81 [citado el 6 de mayo de 2020].

16.- Zhang C, Wu Z, Li J-W, Zhao H, Wang G-Q. Cytokine release syndrome in severe COVID-19: interleukin-6 receptor antagonist tocilizumab may be the key to reduce mortality. Int J Antimicrob Agents [Internet]. 2020; 55 (5): 105954. Available from: https://linkinghub.elsevier.com/retrieve/ pii/S0924857920301047 [citado el 6 de mayo de 2020].

17.- Vaduganathan $M$, Vardeny $O$, Michel $T$, McMurray J J V, Pfeffer M A, Solomon S D. Renin-angiotensin-aldosterone system inhibitors in patients with Covid-19. N Engl J Med [Internet]. 2020; 382(17): 16539. Available from: http://www.nejm.org/ doi/10.1056/NEJMsr2005760 [citado el 6 de mayo de 2020].

18.- Tay M Z, Poh C M, Rénia L, MacAry P A, Ng L F P. The trinity of COVID-19: immunity, inflammation and intervention. Nat Rev Immunol [Internet]. 2020; 1-12. Available from: http://www.nature.com/articles/s41577020-0311-8 [citado el 12 de mayo de 2020].

19.- Mehta N, Kalra A, Nowacki A S, Anjewierden S, Han Z, Bhat P, et al. Association of use of angiotensin-converting enzyme inhibitors and angiotensin ii receptor blockers with testing positive for coronavirus disease 2019 (COVID-19). JAMA Cardiol [Internet]. 2020; Available from: http://www.ncbi.nlm.nih.gov/ pubmed/32369097 [citado el 12 de mayo de
2020].

20.- Bunyavanich S, Do A, Vicencio A. Nasal gene expression of angiotensin-converting enzyme 2 in children and adults. JAMA [Internet]. 2020 May 20; Available from: https://doi. org/10.1001/jama.2020.8707.

21.- Modes of transmission of virus causing COVID-19: implications for IPC precaution recommendations [Internet]. Available from: https://www.who.int/news-room/commentaries/ detail/modes-of-transmission-of-virus-causingcovid-19-implications-for-ipc-precautionrecommendations [citado el 6 de mayo de 2020].

22.- Morawska L, Cao J. Airborne transmission of SARS-CoV-2: The world should face the reality. Environ Int [Internet]. 2020 Jun; 139: 105730. Available from: http:// www.sciencedirect.com/science/article/pii/ S016041202031254X

23.- Xie M, Chen Q. Insight into 2019 novel coronavirus-An updated interim review and lessons from SARS-CoV and MERS-CoV. Int J Infect Dis [Internet]. 2020; 94: 119-24. Available from: https://linkinghub.elsevier.com/ retrieve/pii/S1201971220302046 [citado el 6 de mayo de 2020]

24.- Wu Y, Guo C, Tang L, Hong Z, Zhou J, Dong $X$, et al. Prolonged presence of SARSCoV-2 viral RNA in faecal samples. Lancet Gastroenterol Hepatol [Internet]. 2020; 5 (5): 434-5. Available from: https://linkinghub. elsevier.com/retrieve/pii/S2468125320300832 [citado el 6 de mayo de 2020]

25.- van Doremalen N, Bushmaker T, Morris D H, Holbrook M G, Gamble A, Williamson $\mathrm{BN}$, et al. Aerosol and surface stability of SARS-CoV-2 as compared with SARS-CoV-1. N Engl J Med [Internet]. 2020; 382(16): 1564-7. Available from: http://www.nejm.org/ doi/10.1056/NEJMc2004973 [citado el 6 de mayo de 2020].

26.- Kampf G. Potential role of inanimate surfaces for the spread of coronaviruses and their inactivation with disinfectant agents. Infect Prev Pract [Internet]. 2020; 2 (2): 100044. Available from: https://linkinghub.elsevier.com/ retrieve/pii/S2590088920300081 [citado el 6 de mayo de 2020].

27.- Lauer S A, Grantz K H, Bi Q, Jones F K, Zheng $\mathrm{Q}$, Meredith H R, et al. The incubation period of coronavirus disease 2019 (COVID-19) from publicly reported confirmed cases: estimation and application. Ann Intern Med [Internet]. 2020; 172 (9): 577-82. Available from: https:// www.acpjournals.org/doi/10.7326/M20-0504 [citado el 6 de mayo de 2020].

28.- Qifang B, Yongsheng W, Shujiang M, Chenfei Y, Xuan Z, Zhen Z, et al. Epidemiology and transmission of COVID-19 in Shenzhen China: Analysis of 391 cases and 1,286 of their close contacts. medRxiv [Internet]. 2020; 1-22. Available from: https://www.medrxiv.org/conte $\mathrm{nt} / 10.1101 / 2020.03 .03 .20028423 \mathrm{v} 3$.
29.- Aylward, Bruce (WHO); Liang W (PRC). Report of the WHO-China Joint Mission on Coronavirus Disease 2019 (COVID-19). WHO-China Jt Mission Coronavirus Dis 2019 [Internet]. 2020; 2019 (February): 16-24. Available from: https://www.who.int/docs/ default-source/coronaviruse/who-china-jointmission-on-covid-19-final-report.pdf.

30.- Zou L, Ruan F, Huang M, Liang L, Huang H, Hong Z, et al. SARS-CoV-2 Viral load in upper respiratory specimens of infected patients. N Engl J Med [Internet]. 2020; 382 (12): 1177-9. Available from: http://www.nejm.org/ doi/10.1056/NEJMc2001737 [citado el 6 de mayo de 2020].

31.- Xing Y, Mo P, Xiao Y, Zhao O, Zhang Y, Wang F. Post-discharge surveillance and positive virus detection in two medical staff recovered from coronavirus disease 2019 (COVID-19), China, January to February 2020. Eurosurveillance [Internet]. 2020; 25 (10): 2000191. Available from: https://www.eurosurveillance. org/content/10.2807/1560-7917. ES.2020.25.10.2000191 [citado el 6 de mayo de 2020].

32.- Sethuraman N, Jeremiah SS, Ryo A. Interpreting diagnostic tests for SARS-CoV-2. JAMA [Internet]. 2020; Available from: https://jamanetwork.com/journals/jama/ fullarticle/2765837 [citado el 6 de mayo de 2020].

33.- Guan W, Ni Z, Hu Y, Liang W, Ou C, He J, et al. Clinical characteristics of coronavirus disease 2019 in China. N Engl J Med [Internet]. 2020; 382 (18): 1708-20. Available from: http:// www.nejm.org/doi/10.1056/NEJMoa2002032 [citado el 6 de mayo de 2020].

34.- Chen N, Zhou M, Dong X, Qu J, Gong F, Han Y, et al. Epidemiological and clinical characteristics of 99 cases of 2019 novel coronavirus pneumonia in Wuhan, China: a descriptive study. Lancet [Internet]. 2020; 395 (10223): 507-13. Available from: https://linkinghub.elsevier.com/retrieve/pii/ S0140673620302117 [citado el 6 de mayo de 2020].

35.- Bialek S, Boundy E, Bowen V, Chow N, Cohn A, Dowling N, et al. Severe outcomes among patients with coronavirus disease 2019 (COVID-19) - United States, February 12-March 16, 2020. MMWR Morb Mortal Wkly Rep [Internet]. 2020; 69 (12): 3436. Available from: http://www.cdc.gov/ $\mathrm{mmwr} / \mathrm{volumes} / 69 / \mathrm{wr} / \mathrm{mm} 6912 \mathrm{e} 2 . \mathrm{htm}$ ? cid $=\mathrm{mm} 6912 \mathrm{e} 2 \mathrm{w}$.

36.- Siddiqi H K, Mehra M R. COVID-19 illness in native and immunosuppressed states: A clinical-therapeutic staging proposal. J Heart Lung Transplant [Internet]. 2020; 39 (5): 405-7. Available from: https://linkinghub.elsevier.com/ retrieve/pii/S105324982031473X [citado el 6 de mayo de 2020].

37.- Keyhan S O, Fallahi H R, Cheshmi B. Dysosmia and dysgeusia due to the 2019 novel 
coronavirus; a hypothesis that needs further investigation. Maxillofac Plast Reconstr Surg [Internet]. 2020; 42 (1): 9. Available from: https://jkamprs.springeropen.com/ articles/10.1186/s40902-020-00254-7 [citado el 6 de mayo de 2020].

38.- Spinato G, Fabbris C, Polesel J, Cazzador D, Borsetto D, Hopkins C, et al. Alterations in smell or taste in mildly symptomatic outpatients with SARS-CoV-2 infection. JAMA [Internet]. 2020; Available from: https://jamanetwork.com/ journals/jama/fullarticle/2765183 [citado el 12 de mayo de 2020].

39.- McGonagle D, O’Donnell J, Sharif K, Emery $\mathrm{P}$, Bridgewood C. Immune mechanisms of pulmonary intravascular coagulopathy (PIC) in COVID-19 pneumonia. Lancet Rheumatol [Internet]. 2020; 2019 (20): 1-9. Available from: https://www.researchgate.net/ publication/340621484_Why_the_Immune Mechanisms_of_Pulmonary_Intravascular Coagulopathy_in_COVID-19_Pneumonia are_Distinct_from_Macrophage_Activation_ Syndrome_with_Disseminated_Intravascular Coagulation.

40.- Lala A, Johnson K W, Russak A J, Paranjpe I, Zhao S, Solani S, et al. Prevalence and impact of myocardial injury in patients hospitalized with COVID-19 infection. medRxiv [Internet]. 2020; 2020.04.20.20072702. Available from: http://medrxiv.org/content/ear ly/2020/04/27/2020.04.20.20072702.abstract.

41.- Wu P, Duan F, Luo C, Liu Q, Qu X, Liang L, et al. Characteristics of ocular findings of patients with coronavirus disease 2019 (COVID-19) in Hubei Province, China. JAMA Ophthalmol [Internet]. 2020; 138 (5): 575. Available from: https://jamanetwork.com/journals/ jamaophthalmology/fullarticle/2764083.

42. Mao L, Jin H, Wang M, Hu Y, Chen S, He Q, et al. Neurologic manifestations of hospitalized patients with coronavirus disease 2019 in Wuhan, China. JAMA Neurol [Internet]. 2020 Jun 1; 77(6): 683. Available from: https://doi. org/10.1001/jamaneurol.2020.1127

43. Zubair AS, McAlpine LS, Gardin T, Farhadian S, Kuruvilla DE, Spudich S. Neuropathogenesis and neurologic manifestations of the coronaviruses in the age of coronavirus disease 2019. JAMA Neurol [Internet]. 2020 May 29. Available from: https://doi.org/10.1001/ jamaneurol.2020.2065

44. Cantuti-Castelvetri L, Ohja R, Pedro L, Djannatian M, Franz J, Kuivanen S, et al. Neuropilin-1 facilitates SARS-CoV-2 cell entry and provides a possible pathway into the central nervous system. bioRxiv [Internet]. 2020 Jan 1;2020.06.07.137802. Available from: http://biorxiv.org/content/ear ly/2020/06/10/2020.06.07.137802.abstract

45.- Recalcati S. Cutaneous manifestations in COVID-19: a first perspective. J Eur Acad Dermatology Venereol [Internet]. 2020; 34 (5). Available from: http://doi.wiley.com/10.1111/ jdv.16387 [citado el 6 de mayo de 2020].

46.- Galván Casas C, Català A, Carretero Hernández G, Rodríguez-Jiménez P, Fernández Nieto D, Rodríguez-Villa Lario A, et al. Classification of the cutaneous manifestations of COVID-19: a rapid prospective nationwide consensus study in Spain with 375 cases. Br J Dermatol [Internet]. 2020; bjd.19163. Available from: https://onlinelibrary.wiley.com/doi/abs/10.1111/ bjd.19163.

47.- Castagnoli R, Votto M, Licari A, Brambilla I, Bruno R, Perlini S, et al. Severe Acute Respiratory Syndrome Coronavirus 2 (SARSCoV-2) infection in children and adolescents. JAMA Pediatr [Internet]. 2020; 2. Available from: https://jamanetwork.com/journals/ jamapediatrics/fullarticle/2765169.

48.- Qiu H, Wu J, Hong L, Luo Y, Song Q, Chen D. Clinical and epidemiological features of 36 children with coronavirus disease 2019 (COVID-19) in Zhejiang, China: an observational cohort study. Lancet Infect Dis [Internet]. 2020; 2019 (20): 1-8. Available from: http://dx.doi.org/10.1016/S14733099(20)30198-5.

49.- Xia W, Shao J, Guo Y, Peng X, Li Z, Hu D. Clinical and CT features in pediatric patients with COVID-19 infection: Different points from adults. Pediatr Pulmonol [Internet]. 2020; 55 (5): 1169-74. Available from: https:// onlinelibrary.wiley.com/doi/abs/10.1002/ ppul.24718.

50.- Riphagen S, Gómez X, González-Martínez C, Wilkinson N, Theocharis P. Hyperinflammatory shock in children during COVID-19 pandemic. Lancet [Internet]. 2020; 395 (10237): 1607-8. Available from: http://www.ncbi.nlm.nih.gov/ pubmed/32386565.

51.- Jones VG, Mills M, Suárez D, Hogan C A, Yeh D, Bradley Segal J, et al. COVID-19 and Kawasaki Disease: novel virus and novel case. Hosp Pediatr [Internet]. 2020; Available from: https://hosppeds.aappublications.org/content/ early/2020/04/06/hpeds.2020-0123.long.

52.- Information for Laboratories: 2019nCoV | CDC [Internet]. Available from: https:/www.cdc.gov/coronavirus/2019ncov/lab/index.html?CDC_AA refVal $=$ https $\% 3 \mathrm{~A} \% 2 \mathrm{~F} \% 2 \mathrm{Fwww}_{\text {. }}$. cdc.gov $\% 2$ Fcoronavirus $\% 2 F 2019$ ncov $\% 2$ Flab\%2Frt-pcr-detection-instructions. html [citado el 6 de mayo de 2020].

53.- Zhou F, Yu T, Du R, Fan G, Liu Y, Liu Z, et al. Clinical course and risk factors for mortality of adult inpatients with COVID-19 in Wuhan, China: a retrospective cohort study. Lancet [Internet]. 2020; 395 (10229): 1054-62. Available from: https://inkinghub.elsevier.com/ retrieve/pii/S0140673620305663 [citado el 12 de mayo de 2020].

54.- Wilson N M, Norton A, Young F P, Collins D W. Airborne transmission of severe acute respiratory syndrome coronavirus-2 to healthcare workers: a narrative review.
Anaesthesia [Internet]. 2020; (April): anae.15093. Available from: https:// onlinelibrary.wiley.com/doi/abs/10.1111/ anae. 15093 .

55.- Advice on the use of point-of-care immunodiagnostic tests for COVID-19 [Internet]. Available from: https://www.who.int/ news-room/commentaries/detail/advice-on-theuse-of-point-of-care-immunodiagnostic-testsfor-covid-19 [citado el 6 de mayo de 2020].

56.- Bao L, Deng W, Gao H, Xiao C, Liu J, Xue J, et al. Reinfection could not occur in SARS-CoV-2 infected rhesus macaques. bioRxiv [Internet]. 2020; 2020.03.13.990226. Available from: http://biorxiv.org/content/ear ly/2020/03/14/2020.03.13.990226.abstract.

57.- Kirkcaldy R D, King B A, Brooks J T. COVID-19 and postinfection immunity. JAMA [Internet]. 2020; Available from: https://doi. org/10.1001/jama.2020.7869.

58.- Chandrashekar A, Liu J, Martinot A J, McMahan K, Mercado N B, Peter L, et al. SARS-CoV-2 infection protects against rechallenge in rhesus macaques. Science (80) [Internet]. 2020; 4776 (May): eabc4776. Available from: http://www.ncbi.nlm.nih.gov/ pubmed/32434946.

59.- Culp W C. Coronavirus Disease 2019. A A Pract [Internet]. 2020; 14 (6): e01218. Available from: http://journals.lww.com/10.1213/ XAA.0000000000001218.

60.- Ai T, Yang Z, Hou H, Zhan C, Chen C, Lv W, et al. Correlation of chest CT and RT-PCR testing in coronavirus disease 2019 (COVID-19) in China: a report of 1014 cases. Radiology [Internet]. 2020; 200642. Available from: http:// pubs.rsna.org/doi/10.1148/radiol.2020200642 [citado el 6 de mayo de 2020].

61.- Xing C, Li Q, Du H, Kang W, Lian J, Yuan L. Lung ultrasound findings in patients with COVID-19 pneumonia. Crit Care [Internet]. 2020; 24 (1): 174. Available from: https:// ccforum.biomedcentral.com/articles/10.1186/ s13054-020-02876-9.

62.- Huang C, Wang Y, Li X, Ren L, Zhao J, Hu $\mathrm{Y}$, et al. Clinical features of patients infected with 2019 novel coronavirus in Wuhan, China. Lancet [Internet]. 2020; 395 (10223): 497-506. Available from: https://linkinghub.elsevier.com/ retrieve/pii/S0140673620301835 [citado el 6 de mayo de 2020].

63.- Gandhi R T, Lynch J B, del Río C. Mild or moderate Covid-19. Solomon CG, Editor. N Engl J Med [Internet]. 2020; NEJMcp2009249. Available from: http://www.nejm.org/ doi/10.1056/NEJMcp2009249 [citado el 6 de mayo de 2020].

64.- Gattinoni L, Chiumello D, Caironi P, Busana M, Romitti F, Brazzi L, et al. COVID-19 pneumonia: different respiratory treatments for different phenotypes? Intensive Care Med [Internet]. 2020; 1-4. Available from: http:// link.springer.com/10.1007/s00134-020-06033-2 [citado el 6 de mayo de 2020]. 